\title{
ALGEBRAIC CYCLES AND EVEN UNIMODULAR LATTICES
}

\author{
NEIL DUMMIGAN
}

The number (up to isomorphism) of positive-definite, even, unimodular lattices of rank $8 r$ grows rapidly with $r$. However, Bannai [1] has shown that, when counted according to weight, those with non-trivial automorphisms make up a fraction of the whole, which goes rapidly to zero as $r \rightarrow \infty$. Therefore it is of some interest to produce families of positive-definite, even, unimodular lattices with large automorphism groups and unbounded ranks.

Suppose that $G$ is a finite group and $V$ is an irreducible $\mathbb{Q}[G]$-module such that $V \otimes \mathbb{R}$ is still irreducible. Then, as observed by Gross [8], the space of $G$-invariant symmetric bilinear forms on $V$ is one-dimensional and is necessarily generated by a positive-definite form, unique up to scaling by non-zero positive rationals. Thompson [23] showed that, if $V$ is also irreducible $\bmod p$ for all primes $p$, then it contains an invariant lattice (unique up to scaling) which is even and unimodular with appropriate scaling of the quadratic form. Examples arising in this manner are the $E_{8}$-lattice of rank 8, the Leech lattice of rank 24 and the Thompson-Smith lattice of rank 248. Gow [6] has also constructed some examples associated with the basic spin representations of $2 A_{n}$ and $2 S_{n}$.

Gross [8] showed how one may obtain more examples by generalising Thompson's method to deal with the possibility that $V$ is not absolutely irreducible. Thus he reconstructed the Barnes-Wall lattices of ranks $2^{f+1}$ (which are unimodular when $f$ is even). He also obtained families of lattices associated with the Weil representations of finite symplectic groups (some of which had previously been constructed by Gow [7]) and families of even unimodular lattices of ranks $2 p^{2}\left(p^{2}-1\right)$ associated with the cuspidal unipotent representation of the finite projective unitary group $\mathrm{PU}\left(3, p^{2}\right)$. Tiep $[\mathbf{2 5}, \mathbf{2 6}]$ has investigated further examples of the 'globally irreducible' representations which give rise to special lattices in this way.

The examples considered by Gross are closely related to the Mordell-Weil lattices of Elkies and Shioda $[\mathbf{4 , 2 0}$. These are the groups of rational points on certain constant (or potentially constant) elliptic curves over the function fields of algebraic curves over finite fields. The finite group action comes from automorphisms of the algebraic curve and the quadratic form comes from a scaling of the canonical height pairing.

Elkies [5] has constructed the Barnes-Wall lattices as sublattices of certain Mordell-Weil lattices arising from hyperelliptic curves in characteristic 2 (and the square of the index is the order of the Shafarevich-Tate group). In [3] we showed how the symplectic group lattices may be viewed as sublattices of Mordell-Weil lattices arising from hyperelliptic curves in odd characteristic, thus obtaining a lower bound for the minimal norm.

Received 12 June 1995; revised 20 February 1997.

1991 Mathematics Subject Classification 11G25.

J. London Math. Soc. (2) 56 (1997) 209-221 
The purpose of this paper is to generalise the families of invariant even unimodular lattices associated with $\mathrm{PU}\left(3, p^{2}\right)$ to the case $G:=\mathrm{PU}(n+2, q)$ for any odd $n \geqslant 1$ and any even power $q=p^{f}$ of a prime number; $G$ acts naturally by automorphisms defined over $\mathbb{F}_{q^{2}}$ on the $n$-dimensional projective hypersurface

$$
X_{n}: x_{0}^{q+1}+x_{1}^{q+1}+\cdots+x_{n+1}^{q+1}=0 .
$$

We construct the desired lattices as sublattices of the groups of primitive codimension $(n+1) / 2$ algebraic cycles (modulo numerical equivalence) on $X_{n} \times E$, where the $E$ are elliptic curves chosen in a suitable isogeny class. The rank is $2\left(q^{n+1}-q^{n}+\cdots+q^{2}-q\right)$ and the quadratic form comes from a scaling of the intersection pairing. That it is a definite quadratic form follows from the observation of Gross mentioned in the second paragraph. Notice that this provides examples for which Grothendieck's 'Hodge-style' standard conjecture [9] is true. Similarly the conjecture is true for codimension $n / 2$ cycles on $X_{n}$ when $n$ is even. These $X_{n}$ were also used by Tate [21] as examples for which his conjecture on algebraic cycles and poles of zeta functions may be confirmed.

When $n=1$ these lattices are sublattices of the familiar Mordell-Weil lattices arising from Fermat curves of degree $q+1$. When $n=1$ and $f=2$ they are the examples of Gross already mentioned, but when $n=1$ and $f>2$ they do not come from globally irreducible representations of any groups. Tiep has explained to me how this follows from his work $[\mathbf{2 4}, \mathbf{2 6}]$. (See $[\mathbf{3}$, Section 10].)

\section{Contents}

1. Fermat hypersurfaces and finite unitary groups

2. The definite intersection form

3. Almost global irreducibility

4. The crystalline cohomology of $X_{n}$

5. The range of the cycle class map

6. An even unimodular sublattice

7. Invariance of the sublattice

8. Some remarks

\section{Fermat hypersurfaces and finite unitary groups}

Let $p$ be any prime and $q=p^{f}$ a power of $p$. Let $k$ be the finite field $\mathbb{F}_{q^{2}}$ and let $\bar{k}$ be an algebraic closure. For each $n \geqslant 1$ one may consider the $n$-dimensional Fermat hypersurface of degree $q+1$ in $\mathbb{P}^{n+1} / k$. Then

$$
X_{n}: x_{0}^{q+1}+x_{1}^{q+1}+\cdots+x_{n+1}^{q+1}=0
$$

is its equation in homogeneous coordinates. One checks immediately that $X_{n}$ is nonsingular.

The group $\operatorname{GL}\left(n+2, q^{2}\right)$ of all invertible $(n+2) \times(n+2)$ matrices over $k$ acts on $\mathbb{P}^{n+1} / k$ by its natural action on homogeneous coordinates. We may define a finite unitary group $U(n+2, q)$ as follows:

$$
U(n+2, q)=\left\{A \in \mathrm{GL}\left(n+2, q^{2}\right): A^{(q) t} A=I\right\} .
$$

Here $I$ is the identity matrix, $A^{(q)}$ is the matrix obtained from $A$ by applying the nontrivial automorphism of $k / \mathbb{F}_{q}$ to all entries, and $B^{t}$ denotes the transpose of a matrix $B$. While $\operatorname{GL}\left(n+2, q^{2}\right)$ acts on the projective space $\mathbb{P}^{n+1} / k$ the subgroup $U(n+2, q)$ 
preserves the hypersurface $X_{n}$. Since the subgroup of scalar matrices acts trivially on projective space we mod out by it to obtain an action of the finite projective unitary group $G:=\mathrm{PU}(n+2, q)$ on $X_{n}$. Although $X_{n}$ is clearly defined over $\mathbb{F}_{p}$ we generally consider it as a variety over $k=\mathbb{F}_{q^{2}}$ since $G$ acts on $X_{n}$ by automorphisms defined over $k$.

Fix $l$ to be any prime different from $p$. Then one may consider the $l$-adic cohomology groups $H^{i}\left(\overline{X_{n}}, \mathbb{Q}_{l}\right)$, which vanish except for $0 \leqslant i \leqslant 2 n$. Here $\overline{X_{n}}$ is just $X_{n}$ considered as a variety over $\bar{k}$. Each of these groups is a finite-dimensional vector space over the field $\mathbb{Q}_{l}$ of $l$-adic numbers. Let $\beta_{i}$ be the dimension of $H^{i}\left(\overline{X_{n}}, \mathbb{Q}_{l}\right)$.

Proposition 1. We have

(1) $\beta_{i}=0$ if $i$ is odd and $i \neq n$;

(2) $\beta_{i}=1$ if $i$ is even and $i \neq n$;

(3) $\beta_{n}= \begin{cases}q^{n+1}-q^{n}+\cdots+q^{2}-q & \text { if } n \text { is odd } \\ q^{n+1}-q^{n}+\cdots+q^{3}-q^{2}+q+1 & \text { if } n \text { is even. }\end{cases}$

This is obtained by comparison with characteristic zero and by using well-known results about the Betti numbers of diagonal hypersurfaces [14].

The $q^{2}$-power geometric Frobenius morphism $\phi$ acts on $\overline{X_{n}}$ and hence, by functoriality, on each $H^{i}\left(\overline{X_{n}}, \mathbb{Q}_{l}\right)$. By counting $k$-rational points on $X_{n}$ as in [28] and using the $l$-adic cohomological formula for $\# X_{n}(k)$ one obtains the following.

Proposition 2. The morphism $\phi$ acts on $H^{n}\left(\overline{X_{n}}, \mathbb{Q}_{l}\right)$ as the scalar $(-q)^{n}$.

Let $E / k$ be an elliptic curve such that $\phi$ is the multiplication map [ $-q]$. It is known that such an $E$ may be chosen and is unique up to isogeny over $k$. Further, all the endormorphisms of $E$ are defined over $k$ and the endomorphism ring is isomorphic to some maximal order $R$ (strictly speaking, any of a conjugacy class of isomorphic maximal orders) in $K$, the definite quaternion algebra over $\mathbb{Q}$ ramified at $p$ and $\infty$. For each maximal order $R$ there are one or two possible choices of $E$ in the isogeny class according as the two-sided ideal class group of $R$ is trivial or not. See [27] for details. Observe that $E$ is supersingular. For each $q$ we imagine that a choice of $E$ has been fixed.

\section{The definite intersection form}

Henceforth we suppose that $n$ is odd. Let $Y_{n}=X_{n} \times E$ so $Y_{n}$ is an $(n+1)$ dimensional proper smooth variety defined over $k$. Let $m=(n+1) / 2$. Let $A^{m}$ be the free abelian group generated formally by closed irreducible subvarieties, defined over $k$, of codimension $m$ in $Y_{n}$. Let $B^{m}=A^{m} \otimes_{\mathbb{Z}} \mathbb{Q}$. There is a $\mathbb{Q}$-linear cycle-class map (choosing an identification of $\mathbb{Q}_{l} / \mathbb{Z}_{l}$ with the $l$-power roots of unity)

$$
c: B^{m} \longrightarrow H^{2 m}\left(\overline{Y_{n}}, \mathbb{Q}_{l}\right) .
$$

By the Künneth formula we have

$$
\begin{aligned}
& H^{2 m}\left(\overline{Y_{n}}, \mathbb{Q}_{l}\right) \simeq H^{n}\left(\overline{X_{n}}, \mathbb{Q}_{l}\right) \otimes H^{1}\left(\bar{E}, \mathbb{Q}_{l}\right) \oplus H^{n+1}\left(\overline{X_{n}}, \mathbb{Q}_{l}\right) \otimes H^{0}\left(\bar{E}, \mathbb{Q}_{l}\right) \\
& \oplus H^{n-1}\left(\overline{X_{n}}, \mathbb{Q}_{l}\right) \otimes H^{2}\left(\bar{E}, \mathbb{Q}_{l}\right) .
\end{aligned}
$$

There is a bilinear intersection pairing $\cdot: A^{m} \times A^{m} \rightarrow \mathbb{Z}$ which extends to $: B^{m} \times B^{m}$ $\rightarrow \mathbb{Q}$. There is also the cup-product $\cdot: H^{2 m}\left(\overline{Y_{n}}, \mathbb{Q}_{l}\right) \times H^{2 m}\left(\overline{Y_{n}}, \mathbb{Q}_{l}\right) \rightarrow \mathbb{Q}_{l}$. We use the 
same notation twice without risk of confusion, particularly since intersection and cup-products are compatible: for any $b_{1}, b_{2} \in B^{m}$ we have $b_{1} \cdot b_{2}=c\left(b_{1}\right) \cdot c\left(b_{2}\right)$. An element $b \in B^{m}$ is said to be $l$-cohomologically equivalent to zero if $c(b)=0$. It is said to be numerically equivalent to zero if $b \cdot b^{\prime}=0$ for all $b^{\prime} \in B^{m}$. The above compatibility clearly implies that if a cycle is $l$-cohomologically equivalent to zero then it is numerically equivalent to zero. We denote this $B_{l}^{m} \subset B_{\text {num }}^{m}$.

The $q^{2}$-power geometric Frobenius morphism $\phi$ acts on $H^{2 m}\left(\overline{Y_{n}}, \mathbb{Q}_{l}\right)$ as the scalar $q^{n+1}$. A conjecture of Tate $[\mathbf{2 1}]$ then predicts that $c\left(B^{m}\right) \mathbb{Q}_{l}=H^{2 m}\left(\bar{Y}_{n}, \mathbb{Q}_{l}\right)$.

Proposition 3. We have

(1) $c\left(B^{m}\right) \mathbb{Q}_{l}=H^{2 m}\left(\overline{Y_{n}}, \mathbb{Q}_{l}\right)$,

(2) $B_{l}^{m}=B_{\text {num }}^{m}$,

(3) $c$ induces an isomorphism $\left(B^{m} / B_{\text {num }}^{m}\right) \otimes \mathbb{Q}_{l} \simeq H^{2 m}\left(\overline{Y_{n}}, \mathbb{Q}_{l}\right)$.

Proof. One may prove (1) by directly imitating the argument of Katsura and Shioda [13, Section 2] using Tate's theorem on homomorphisms of abelian varieties over finite fields [22] and the inductive structure of Fermat varieties. Given this, (2) and (3) follow from Proposition 8.4 of [18].

Recall that $H^{2 m}\left(\overline{Y_{m}}, \mathbb{Q}_{l}\right)$ is a direct sum of three summands, listed above. The second and third summands are each one-dimensional, generated by the classes of cycles $x, y \in B^{m}$ respectively; $x$ may be taken to be the product with $E$ of the intersection of $m$ hyperplane sections of $X_{n}$ and $y$ may be taken to be the product with a point of $E$ of the intersection of $m-1$ hyperplane sections of $X_{n}$. Then $x \cdot x=y \cdot y=0$ and $x \cdot y \neq 0$. Define

$$
C^{m}=\left\{b \in B^{m}: b \cdot x=b \cdot y=0\right\} .
$$

Since the first summand of $H^{2 m}\left(\bar{Y}_{n}, \mathbb{Q}_{l}\right)$ is clearly orthogonal to the other two with respect to cup-product, we find that

$$
c\left(C^{m}\right) \mathbb{Q}_{l}=H^{n}\left(\overline{X_{n}}, \mathbb{Q}_{l}\right) \otimes H^{1}\left(\bar{E}, \mathbb{Q}_{l}\right) .
$$

Definition 1. $V=C^{m} /\left(C^{m} \cap B_{\text {num }}^{m}\right)$.

Thus $V$ is a $\mathbb{Q}$-vector space of dimension $2\left(q^{n+1}-q^{n}+\cdots+q^{2}-q\right)$. We have

$$
V \otimes \mathbb{Q}_{l} \simeq H^{n}\left(\overline{X_{n}}, \mathbb{Q}_{l}\right) \otimes H^{1}\left(\bar{E}, \mathbb{Q}_{l}\right)
$$

Further, the natural action of $G=\mathrm{PU}(n+2, q)$ on $X_{n}$ endows $V$ with the structure of $\mathbb{Q}[G]$-module. The intersection pairing gives us a $G$-invariant symmetric bilinear form $\langle\rangle:, V \times V \rightarrow \mathbb{Q}$. The faithful action of the quaternion algebra $K$ on $H^{1}\left(\bar{E}, \mathbb{Q}_{l}\right)$ commutes with the action of $G$ on $H^{n}\left(\overline{X_{n}}, \mathbb{Q}_{l}\right)$ so $K$ is naturally contained in the endomorphism algebra $\operatorname{End}_{\mathbb{Q}[G]}(V)$.

Proposition 4. We have

(1) $V$ is an irreducible $\mathbb{Q}[G]$-module,

(2) $V \otimes \mathbb{R}$ is an irreducible $\mathbb{R}[G]$-module,

(3) $\langle$,$\rangle is definite.$

Proof. (1) According to [11], the $\mathbb{Q}_{l}[G]$-module $H^{n}\left(\overline{X_{n}}, \mathbb{Q}_{l}\right)$ is absolutely irreducible with character $\psi$, say. It follows that the $\mathbb{Q}[G]$-module $V$ has character $2 \psi$. 
If it were reducible (over $\mathbb{Q}$ ) its endomorphism algebra $\operatorname{End}_{\mathbb{Q}[G]}(V)$ would be $M_{2}(\mathbb{Q})$, which is impossible since we know that it contains (in fact is equal to) the quaternion algebra $K$.

(2) Similarly $V \otimes \mathbb{R}$ is irreducible since $K \otimes \mathbb{R}$, the Hamiltonian quaternion algebra, is not $M_{2}(\mathbb{R})$.

(3) Since $V \otimes \mathbb{R}$ is irreducible it follows from [8, Section 2] that the space of $G$ invariant symmetric bilinear forms on $V \otimes \mathbb{R}$ is one-dimensional and is generated by a positive-definite form. The extension of $\langle$,$\rangle to V \otimes \mathbb{R}$ is $G$-invariant and is not zero (by definition of numerical equivalence) so must be a definite form.

As noted in the introduction, (3) is what is predicted by a conjecture of Grothendieck [9]. Actually, the space of primitive codimension $m$ cycles (modulo numerical equivalence) on $Y_{n}$, with respect to a natural ample divisor, is a little larger than $V$, containing also the class of $x-y$. However, $V$ is the interesting part.

\section{Almost global irreducibility}

Recall that $R$ is a maximal order in $K$ and is the endomorphism ring of $E$. The group ring $R[G]$ acts naturally on $V$ and we let $M$ be some $R[G]$-invariant lattice in $V$. For definiteness we may take $M$ to be the image in $V$ of $A^{m} \cap C^{m}$.

Proposition 5. (1) The character $\psi$ is absolutely irreducible mod $l$ for each prime $l \neq p$.

(2) The quotient $M / l M$ is an irreducible $(R / l R)[G]$-module for each prime $l \neq p$.

(3) After rescaling $\langle$,$\rangle by some non-zero rational number coprime to p$, we may assume that it is integral on $M$ and that $\operatorname{det} M\left(\right.$ that is, $\operatorname{det}\left(\left\langle v_{i}, v_{j}\right\rangle\right)$, where $\left\{v_{i}\right\}$ is a $\mathbb{Z}$-basis for $M$ ) is purely a power of $p$.

Proof. Suppose that $l \neq p$ is a prime such that $\psi$ is not absolutely irreducible $\bmod l$. Then $H^{n}\left(\overline{X_{n}}, \mathbb{Q}_{l}\right)$ provides a representation with coefficients in $\mathbb{Q}_{l}$ affording the character $\psi$. Let $S$ be a $G$-stable $\mathbb{Z}_{l}$-lattice in $H^{n}\left(\overline{X_{n}}, \mathbb{Q}_{l}\right)$. Our supposition (stated more precisely) is that the $\mathbb{F}_{l}[G]$-module $S / l S$ is not absolutely irreducible. Observe that $q^{n+1}-q^{n}+\cdots+q^{2}-q$ is the smallest dimension of an absolutely irreducible, nontrivial representation of $G$ in any characteristic not equal to $p[\mathbf{1 5}]$. Hence the only possibility is that for some sufficiently large $d$ the irreducible composition factors of the $\mathbb{F}_{l^{d}}[G]$-module $(S / l S) \otimes_{\mathbb{F}_{l}} \mathbb{F}_{l^{d}}$ are all trivial. (Recall that if $l \mid \# G$ then representations of $G$ in characteristic $l$ are not necessarily completely reducible.)

Choosing a suitable basis, the action of $G$ on $(S / l S) \otimes \mathbb{F}_{l^{d}}$ is by upper-triangular, unipotent matrices over $\mathbb{F}_{l^{d}}$. Letting $U$ be the group of such matrices, we have a homomorphism $\theta: G \rightarrow U$. Now $\# U$ is a power of $l$ so the restriction of $\theta$ to any subgroup $H$ of $G$ of order prime to $l$ is trivial. Let $S^{\prime}=S \otimes_{\mathbb{Z}_{l}} W\left(\mathbb{F}_{l^{d}}\right)$, where $W\left(\mathbb{F}_{l^{d}}\right)$ is the ring of integers in the unramified extension of degree $d$ of $\mathbb{Q}_{l}$. We have just seen that if $H$ is a subgroup of $G$ of order prime to $l$ than $H$ acts trivially on $S^{\prime} / l S^{\prime}$. By induction and using a similar argument about homomorphisms of $H$ to groups of $l$ power order being trivial, we see that $H$ acts trivially on $S^{\prime} / l^{r} S^{\prime}$ for all $r \geqslant 1$, hence that $H$ acts trivially on $S$ and on $H^{n}\left(\overline{X_{n}}, \mathbb{Q}_{l}\right)$.

Now we make a special choice of $H$. There is a subgroup of $G$ isomorphic to the symmetric group $S_{n+2}$, which acts on $X_{n}$ by permuting the coordinates $x_{0}, x_{1}, \ldots, x_{n+1}$. 
If $l \neq 2$ we let $H$ be the cyclic subgroup generated by some arbitrary fixed transposition. If $l=2$ we use a 3 -cycle instead. It follows from Section 5, Proposition 8, that the crystalline cohomology $H_{\text {crys }}^{n}\left(X_{n}\right)$ provides a representation with coefficients in $W=W\left(\mathbb{F}_{q^{2}}\right)$ affording the character $\psi$. Therefore $H$ acts trivially on $H_{\text {crys }}^{n}\left(X_{n}\right)$ and on its reduction $\bmod p$, the algebraic de Rham cohomology $H_{d R}^{n}\left(X_{n}\right)$. But the next section contains explicit bases for the subfactors of the Hodge filtration of $H_{d R}^{n}\left(X_{n}\right)$ and it is clear that the action of $H$ (permuting coordinates) is not trivial. This contradiction shows that (1) must be true.

(2) follows from (1) and [8, Proposition 4.2(3)]. Then (3) follows from the arguments of Sections 3 and 5 of $[\mathbf{8}]$ (note that $\langle$,$\rangle does come from a G$-invariant $K$-Hermitian form since the one-dimensional space of such forms is identified with the one-dimensional space of $G$-invariant symmetric bilinear forms).

Although $R$ and $M$ depend on the particular choice of $E$, the $\mathbb{Q}[G]$-module $V$ does not, since an irreducible $\mathbb{Q}[G]$-module is determined by its character, and the character $2 \psi$ is determined without reference to $E$ as twice the character of the $\mathbb{Q}_{l}[G]$ module $H^{n}\left(\overline{X_{n}}, \mathbb{Q}_{l}\right)$. By choosing the sign of the scaling factor appropriately, we may assume that $\langle$,$\rangle is positive-definite. Our goal in the rest of the paper is to produce an$ $R[G]$-invariant sublattice of $M$ which, after rescaling $\langle$,$\rangle by a power of p$, is even and unimodular, at least in the case that $f$ is even. To get a suitable model of $V \otimes \mathbb{Q}_{p}$ we need to use crystalline cohomology, for which a good concise reference is [12].

\section{The crystalline cohomology of $X_{n}$}

Let $W$ be the ring of infinite Witt vectors over $k$. Thus $W$ is the ring of integers in the unramified extension $L$ of degree $2 f$ of $\mathbb{Q}_{p}$. Its maximal ideal is $p W$ and $W / p W \simeq k$. Let $\sigma$ be the automorphism of $W / \mathbb{Z}_{p}$ which lifts the $p$ th-power automorphism of $k$. Associated with any proper, smooth variety $Z / k$ of dimension $d$ are finitely generated $W$-modules $H^{i}(Z)=H_{\text {crys }}^{i}(Z)$ which are trivial except for $0 \leqslant i \leqslant 2 d$. The dimension of $H^{i}(Z)$ is equal to the dimension of $H^{i}\left(\bar{Z}, \mathbb{Q}_{l}\right)$ for any prime $l \neq p$. There is a cup-product structure and if $Z$ is a hypersurface then $H^{i}(Z)$ is a free $W$-module. If $Z^{*} / W$ is a proper smooth scheme whose special fiber is $Z / k$, then there is a natural isomorphism $H^{i}(Z) \simeq H_{d R}^{i}\left(Z^{*}\right)$, the $i$ th algebraic de Rham cohomology of $Z^{*}$. We always choose $X_{n}^{*}$ to be the natural lifting of $X_{n}$ defined by the same equation as $X_{n}$. The action of the diagonal subgroup $T$ of $G$ on $X / k$ lifts to $X^{*} / W$ though the action of the whole group $G$ does not.

The identification of $H^{n}\left(X_{n}\right)$ with $H_{d R}^{n}\left(X_{n}^{*}\right)$ endows it with a Hodge filtration

$$
H^{n}\left(X_{n}\right)=M_{0} \supset \ldots \supset M_{n+1}=\{0\},
$$

and for all $0 \leqslant k \leqslant n$ we have $M_{k} / M_{k+1} \simeq H^{n-k}\left(X_{n}^{*}, \Omega^{k}\right)$, the cohomology of the $k$ th exterior power of the sheaf of differentials on $X_{n}^{*}$.

Proposition 6. (1) The cohomology $H^{n-k}\left(X_{n}^{*}, \Omega^{k}\right)$ has a W-basis consisting of elements which may be written

$$
w_{m_{0}, m_{1}, \ldots, m_{n+1}}=x_{0}^{m_{0}} x_{1}^{m_{1}} \ldots x_{n+1}^{m_{n+1}} d x_{0} \ldots d x_{n+1},
$$

where $\left(m_{0}, \ldots, m_{n+1}\right)$ ranges over all $(n+2)$-tuples such that $0 \leqslant m_{i} \leqslant q-1$ for each $i$ and $\sum m_{i}=(n+1-k) q-(k+1)$. 
(2) An element $\operatorname{diag}\left(a_{0}, \ldots, a_{n+1}\right)$ of $T$ acts on $w_{m_{0} \ldots, m_{n+1}}$ as the scalar

$$
a_{0}^{m_{0}+1} \ldots a_{n+1}^{m_{n+1}+1} .
$$

(3) The $w_{n_{0}}$ lift to a $W$-basis for $H^{n}\left(X_{n}\right)$ consisting of simultaneous eigenvectors for $T$ with distinct characters. (We also denote these basis elements $\left.w_{m_{0}, \ldots, m_{n+1}}.\right)$

Proof. (1) follows from [19, Section 3]. Then (2) is obvious. Observing that the characters of $T$ arising are all distinct, (3) then follows from the fact that $p$ does not divide the order of $T$.

DEFINITION 2. If $\sum m_{i}=(n+1-k) q-(k+1)$ we say that $w_{m_{0}, \ldots, m_{n+1}}$ (or any nonzero multiple of it) is of type $k$.

The absolute $p$ th-power Frobenius morphism of the scheme $X_{n}$ to itself (defined to be the identity on points and the $p$ th power on the structure sheaf) gives rise, by functoriality, to a $\sigma$-linear map $F$ from $H^{n}\left(X_{n}\right)$ to itself. Thus $F(a v)=a^{\sigma} F(v)$ for any $a \in W$ and $v \in H^{n}\left(X_{n}\right)$.

Proposition 7. (1) If $w$ is one of our standard basis elements for $H^{n}\left(X_{n}\right)$ then $F w=c w^{\prime}$ for some $c \in W$ and $w^{\prime}$ some standard basis element. The character of $T$ associated to $w^{\prime}$ is $p$ times that associated to $w$.

(2) If $w$ is of type $k$ then $\operatorname{ord}_{p}(c)=k$.

(3) If $w$ is of type $k$ then $F^{f} w$ is of type $n-k$.

Proof. (1) For any fixed $t \in T$ suppose that $t . w=\lambda w$. Then $t . F w=F(t . w)=$ $F(\lambda w)=\lambda^{\sigma} F w=\lambda^{p} F w$ since $\lambda$ is a root of unity. Thus $F w$ is a simultaneous eigenvector for $T$, its associated character being $p$ times that for $w$. It must be a multiple of one of our standard basis of elements since their associated characters are all distinct, c.f. $[10,19,20]$.

(2) Ogus explains near the end of this paper [19] how this follows from Mazur's work on Frobenius and the Hodge filtration $[\mathbf{1 6}, \mathbf{1 7}]$.

(3) By (1), $F^{f}$ multiplies characters by $p^{f}=q$ but $T$ has exponent $q+1$ so $F^{f}$ multiplies characters by -1 . Since $\left(q-1-m_{i}\right)+1=(q+1)-\left(m_{i}+1\right)$, one sees that

$$
w_{q-1-m_{0}, \ldots, q-1-m_{n+1}}
$$

has minus the character of $w_{m_{0}, \ldots, m_{n+1}}$. If $\sum m_{i}=(n+1-k) q-(k+1)$ one easily checks that $\sum\left(q-1-m_{j}\right)=(n+1-(n-k)) q-((n-k)+1)$.

Adopting a new notation, suppose that $w_{1}$ is some standard basis element in $H^{n}\left(X_{n}\right)$. Then there are standard basis elements $w_{i}$ for $i \geqslant 1$ and elements $c_{i} \in W$ such that $F w_{i}=c_{i} w_{i+1}$ for each $i$ and $\operatorname{ord}_{p}\left(c_{i}\right)=k_{i}$, where $w_{i}$ is of type $k_{i}$. It follows from the crystalline cohomological formula for $\# X_{n}(k)$ that $F^{2 f}$ acts as multiplication by $(-q)^{n}$ on $H^{n}\left(X_{n}\right)$. Hence $w_{i+2 f}=w_{i}$ and subscripts may be thought of $\bmod 2 f$. We get cyclic ' $F$-orbits' of basis elements of lengths dividing $2 f$. Note that $k_{i}+k_{f+i}=n$. Incidentally, it follows fairly easily from the fact that $X_{n}$ is really defined over $\mathbb{F}_{p}$ that all the $c_{i}$ are actually in $\mathbb{Z}_{p}$. 


\section{The range of the cycle class map}

Let $c: B^{m} \rightarrow H^{2 m}\left(Y_{n}\right) \otimes_{W} L$ be the cycle map constructed by Milne in Section 2 of [18]. Then $c$ is compatible with intersection and cup products and $c\left(B^{m}\right) \subset$ $H^{2 m}\left(Y_{n}\right)^{F=p^{m}} \otimes_{\mathbb{Z}_{p}} \mathbb{Q}_{p}$. As for $l$-adic cohomology, there is a Künneth formula (use [12, 3.3] and the fact that everything is torsion-free) and $c\left(C^{m}\right) \subset H^{n}\left(X_{n}\right) \otimes H^{1}(E) \otimes L$, so that $c\left(C^{m}\right) \subset\left(H^{n}\left(X_{n}\right) \otimes H^{1}(E)\right)^{F=p^{m}} \otimes_{\mathbb{Z}_{p}} \mathbb{Q}_{p}$.

Proposition 8. (1) $c\left(C^{m}\right) \mathbb{Q}_{p}=\left(H^{n}\left(X_{n}\right) \otimes H^{1}(E)\right)^{F=p^{m}} \otimes_{\mathbb{Z}_{p}} \mathbb{Q}_{p}$.

(2) $V \otimes_{\mathbb{Q}} \mathbb{Q}_{p} \simeq\left(H^{n}\left(X_{n}\right) \otimes H^{1}(E)\right)^{F=p^{m}} \otimes \mathbb{Q}_{p}$.

Proof. We shall see soon that the dimension of the right-hand side is $2\left(q^{n+1}-q^{n}+\cdots+q^{2}-q\right)$. The action of $K \otimes \mathbb{Q}_{p}$ on $H^{1}(E)$ commutes with $F$, as does the action of $G$ on $H^{n}\left(X_{n}\right)$, so $K \otimes \mathbb{Q}_{p}$ acts by $\mathbb{Q}_{p}[G]$-endomorphisms on the righthand side. Since $K \otimes \mathbb{Q}_{p} \neq M_{2}\left(\mathbb{Q}_{p}\right)$, we find that the right-hand side is an irreducible $\mathbb{Q}_{p}[G]$-module. (The $W[G]$-module $H^{n}\left(X_{n}\right)$ must be absolutely irreducible, nontrivial of minimal degree, since the trivial character of $T$ does not occur.) Now $c\left(C_{m}\right) \mathbb{Q}_{p}$ is a $\mathbb{Q}_{p}[G]$-submodule so is either zero or the whole thing. But $C^{m}$ contains elements which are not numerically equivalent to zero, so $c\left(C^{m}\right) \mathbb{Q}_{p}$ cannot be zero and must be the whole thing. Hence we obtain (1), and (2) now follows by comparing dimensions.

Lemma 1. The cohomology $H^{1}(E)$ has a $W$-basis $\left\{e_{0}, e_{1}\right\}$ such that $F e_{0}=e_{1}$ and $F e_{1}=\gamma e_{0}$ with $\operatorname{ord}_{p}(\gamma)=1$.

For the proof see [2, Lemma 4].

Definition 3. $S:=\left(H^{n}\left(X_{n}\right) \otimes H^{1}(E)\right)^{F=p^{m}}$.

Our next task is to identify this $\mathbb{Z}_{p}$-module. The condition $F=p^{m}$ can be dealt with one $F$-orbit at a time. Given an $F$-orbit $J=\left\{w_{1}, w_{2}, \ldots, w_{2 f}\right\}$ (note that this list may involve repetition) we define $H_{J}$ to be the $W$-submodule of $H^{n}\left(X_{n}\right)$ generated by $J$. Let $S_{J}=S \cap\left(H_{J} \otimes H^{1}(E)\right)$. Then $S=\oplus_{J} S_{J}$ and it suffices to examine each $S_{J}$.

Now $F^{f}$ inverts characters and one may check that no self-inverse character occurs, so $f$ is not divisible by the length of any $F$-orbit. Hence the length of the $F$-orbit $J$ is of the form $2 l$ for some $l \mid f$. Suppose that $w \in S_{J}$, say

$$
w=\sum_{i=1}^{2 l}\left(a_{i} w_{i} \otimes e_{0}+b_{i} w_{i} \otimes e_{1}\right) .
$$

Then $F w=\sum\left(a_{i}^{\sigma} c_{i} w_{i+1} \otimes e_{1}+b_{i}^{\sigma} c_{i} \gamma w_{i+1} \otimes e_{0}\right)$. Since we require $F w=p^{m} w=$ $p^{(n+1) / 2} w$ we have the conditions

hence

$$
\begin{gathered}
a_{i}^{\sigma} c_{i}=p^{m} b_{i+1}, \\
b_{i}^{\sigma} c_{i} \gamma=p^{m} a_{i+1},
\end{gathered}
$$

so

$$
\begin{gathered}
a_{i}^{\sigma^{2}} c_{i} c_{i+1} \gamma=p^{2 m} a_{i+2}=p^{n+1} a_{i+2}, \\
b_{i}^{\sigma^{2}} c_{i} c_{i+1} \gamma^{\sigma}=p^{2 m} b_{i+2}=p^{n+1} b_{i+2},
\end{gathered}
$$

$$
\begin{aligned}
& \operatorname{ord}_{p}\left(a_{i+2}\right)=\operatorname{ord}_{p}\left(a_{i}\right)+k_{i}+k_{i+1}-n, \\
& \operatorname{ord}_{p}\left(b_{i+2}\right)=\operatorname{ord}_{p}\left(b_{i}\right)+k_{i}+k_{i+1}-n .
\end{aligned}
$$

Henceforth we assume that $f$ is even. 
The $F$-orbit $J=\left\{w_{1}, w_{2}, \ldots, w_{2 l}\right\}$ may be divided into two 'complementary' $F^{2}$ orbits $J_{0}=\left\{w_{1}, w_{3}, \ldots, w_{2 l-1}\right\}$ and $J_{e}=\left\{w_{2}, w_{4}, \ldots, w_{2 l}\right\}$. (Here $w_{1}$ has been chosen arbitrarily.) We see from the above equations that the coefficient $a_{1}$ determines all the $a_{i}$ for $i$ odd and all the $b_{i}$ for $i$ even. But a particular choice of $a_{1}$ will not lead to an element of $S_{J}$ unless

$$
a_{1}^{\sigma^{2 l}} c_{1} c_{2} \ldots c_{2 l} \gamma \gamma^{\sigma^{2}} \ldots \gamma^{\sigma^{2 l-2}}=p^{l(n+1)} a_{1} .
$$

Using the fact that $F^{2 f}$ acts as $-q^{n}$ on $H^{n}\left(X_{n}\right)$ and as $-q$ on $H^{1}(E)$, combined with Hilbert's Theorem 90, one easily checks that the possible coefficients $a_{1}$ form a $\mathbb{Z}_{p^{-}}$ submodule of rank $2 l$ of $W$. Similar remarks apply to $b_{1}$. Hence we obtain the following.

Lemma 2. The $\mathbb{Z}_{p}$-module $S_{J}$ is free of rank $4 l$ and $S$ is a free $\mathbb{Z}_{p}$-module of rank twice the $W$-rank of $H^{n}\left(X_{n}\right)$, namely $2\left(q^{n+1}-q^{n}+\cdots+q^{2}-q\right)$.

In order for all the $\operatorname{ord}_{p}\left(a_{i}\right)$ and $\operatorname{ord}_{p}\left(b_{i}\right)$ to be nonnegative there are certain nonnegative integers $n_{i}$ and $m_{i}$ such that necessarily $\operatorname{ord}_{p}\left(a_{i}\right) \geqslant n_{i}$ and $\operatorname{ord}_{p}\left(b_{i}\right) \geqslant m_{i}$. We have

$$
\begin{gathered}
m_{i+1}=n_{i}+k_{i}-m, \\
n_{i+1}=m_{i}+k_{i}+1-m, \\
n_{i+2}=n_{i}+k_{i}+k_{i+1}-n, \\
m_{i+2}=m_{i}+k_{i}+k_{i+1}-n .
\end{gathered}
$$

Then $S_{J} \otimes_{\mathbb{Z}_{p}} W=S_{J} W$ may be decomposed according to characters of $T$ and has a basis

$$
\left\{p^{n_{i}} w_{i} \otimes e_{0}, p^{m_{i}} w_{i} \otimes e_{1}: 1 \leqslant i \leqslant 2 l\right\} .
$$

Lemma 3. (1) For a given F-orbit $J$ the quantity $n_{i}+m_{f+i}$ depends only on $i \bmod 2$.

(2) In fact, $n_{i}+m_{f+i}$ depends only on $J$.

Proof. (1) Using equations (9) and (10) we get

$$
\left(n_{i+2}+m_{f+i+2}\right)-\left(n_{i}+m_{f+i}\right)=k_{i}+k_{f+i}+k_{i+1}+k_{f+i+1}-2 n,
$$

but this is zero since $k_{j}+k_{f+j}=n$ for all $j$.

(2) Recall that $f$ is even. We check that

$$
\begin{aligned}
& \left(n_{i}+m_{f+i}\right)-\left(n_{f+i+1}+m_{i+1}\right)=\left(n_{i}-m_{i+1}\right)+\left(m_{f+i}-n_{f+i+1}\right) \\
& \quad=\left(m-k_{i}\right)+\left(m-1-k_{f+i}\right)=2 m-1-\left(k_{i}+k_{f+i}\right)=n-n=0 .
\end{aligned}
$$

Definition 4. (1) $d_{J}:=n_{i}+m_{f+i}$,

(2) $d_{\max }=\max _{J} d_{J}$,

(3) $r_{J}=d_{\max }-d_{J}$.

\section{An even unimodular sublattice}

The $W$-modules $H^{1}(E)$ and $H^{n}\left(X_{n}\right)$ are self-dual with respect to the cup-product pairings. This is Poincare duality (use $[\mathbf{1 2}, 3.5 .4]$ and the fact that everything is torsion-free). 
Lemma 4. (1) We have $e_{0} \cdot e_{0}=e_{1} \cdot e_{1}=0$ and $e_{0} \cdot e_{1}$ is a unit in $W$.

(2) If we and $w^{\prime}$ are standard basis elements for $H^{n}\left(X_{n}\right)$ then $w \cdot w^{\prime}=0$ unless the characters of $T$ associated to $w$ and $w^{\prime}$ form an inverse pair. If this happens (that is, if $w$ and $w^{\prime}$ belong to the same F-orbit and are of the form $\left.w_{i}, w_{f+i}\right)$ then $w \cdot w^{\prime}$ is a p-adic unit.

Proof. (1) follows from the fact that the cup-product pairing on $H^{1}(E)$ is skewsymmetric and perfect. (2) follows from the fact that the cup-product pairing on $H^{n}\left(X_{n}\right)$ is $T$-invariant and perfect.

Lemma 5. (1) If $J$ is an F-orbit and $v, v^{\prime} \in S_{J}$ then $v \cdot v^{\prime}$ is divisible by $p^{a_{J}}$.

(2) Moreover, $\operatorname{det} S_{J}=p^{d_{J} \text { rank } S_{J}}$. (This is the determinant of the restriction of the cup-product pairing and is well defined as a power of $p$.)

Proof. (1) is an easy consequence of the previous lemma and $\operatorname{det} S_{J}$ may be calculated using our basis for $S_{J} \otimes_{\mathbb{Z}_{p}} W$.

Recall that $R=\operatorname{End}(E)$ is a maximal order in $K$, the definite quaternion algebra over $\mathbb{Q}$ ramified at $p$ and $\infty$. So there is a two-sided maximal ideal $\mathfrak{p}$ of $R$ such that $p R=\mathfrak{p}^{2}$. Choose $\pi \in \mathfrak{p}$ such that $\pi \notin p R$. The action of $R$ on $H^{1}(E)$ commutes with $F$ so $R$ acts on $S$.

LeMma 6. We have

(1) $\operatorname{det} \pi^{r_{J}} S_{J}=p^{d_{\max } \operatorname{rank} S_{J}}$,

(2) up to a p-adic unit, $(\pi v) \cdot\left(\pi v^{\prime}\right)=p v \cdot v^{\prime}$ for all $v, v^{\prime} \in S$,

(3) $v \cdot v^{\prime}$ is divisible by $p^{d_{\max }}$ for all $v, v^{\prime} \in \pi^{r_{J}} S_{J}$.

Proof. (1) follows from (2) of the previous lemma. (3) follows from (2) combined with (1) of the previous lemma. To prove (2) we note that $S \otimes \mathbb{Q}_{p}=V \otimes \mathbb{Q}_{p}$ and cupproduct on the left agrees with $\langle$,$\rangle on the right (up to a p$-adic unit). The symmetric bilinear form such that $\left(v, v^{\prime}\right) \mapsto\left\langle\pi v, \pi v^{\prime}\right\rangle$ for all $v, v^{\prime} \in V$ is $G$-invariant, so is necessarily of the form $\left(v, v^{\prime}\right) \mapsto c .\left\langle v, v^{\prime}\right\rangle$ for some non-zero rational number $c$. Since $\mathfrak{p}^{2}=p R$ we find that $\operatorname{ord}_{p}(c)=1$, as desired.

We define a $\mathbb{Z}_{p}$-submodule $\mathscr{L}$ of $S$ by

$$
\mathscr{L}=\oplus_{J} \pi^{r_{J}} S_{J} .
$$

Then $\mathscr{L}$ is a $\mathbb{Z}_{p}$-lattice in $V \otimes \mathbb{Q}_{p}$. Recall that we have a $\mathbb{Z}$-lattice $M$ in $V$ and that $\langle$,〉 is normalised, so that $M$ is an integral lattice and $\operatorname{det} M$ is a power of $p$.

Definition 5. Let $L$ be the lattice in $V$ such that $L \otimes \mathbb{Z}_{l}=M \otimes \mathbb{Z}_{l}$ for all primes $l \neq p$ but $L \otimes \mathbb{Z}_{p}=\mathscr{L}$.

Proposition 9. (1) For all $v \in L$ we have $\langle v, v\rangle \in 2 p^{d_{\max }} \mathbb{Z}$.

(2) Also, $\operatorname{det} L=p^{d_{\max } \operatorname{rank} L}$.

Proof. (2) follows from (1) of the previous lemma. Suppose that $v \in L$. To show that $\langle v, v\rangle \in 2 \mathbb{Z}_{l}$ for all primes $l \neq p$ just copy the argument in the proof of $[\mathbf{8}$, Proposition 8.3], that is, use the fact that $\langle$,$\rangle is the trace of a Hermitian form. It$ 
remains to show that $\langle v, v\rangle \in 2 p^{d_{\max }} \mathbb{Z}_{p}$. For this recall the basis for $S$ obtained in Section 5. If we express $v$ in terms of this basis and consider $v \cdot v$ we see that only the cross terms survive and each appears twice. Hence we get the factor 2, and the power of $p$ comes from (3) of the previous lemma.

Now we simply rescale $\langle$,$\rangle by dividing it by p^{d_{\max }}$ to obtain $L$ as an even, unimodular lattice in $V$. In other words, $\langle v, v\rangle \in 2 \mathbb{Z}$ for all $v \in L$ and $\operatorname{det} L=1$. Also $L$ is $R$-stable. It remains to prove that it is $G$-invariant.

\section{Invariance of the sublattice}

The group $A^{m}$, of codimension $m$ cycles on $Y_{n}$, is clearly $G$-invariant, so the lattice $M$ in $V$ is $G$-invariant. Moreover, $L \otimes \mathbb{Z}_{l}=M \otimes \mathbb{Z}_{l}$ for all primes $l \neq p$, so it remains to show that $L \otimes \mathbb{Z}_{p}$ is $G$-invariant. Let $J$ and $J^{\prime}$ be two $F$-orbits. Any fixed element $g \in G$ maps $S$ to itself. Restricting the domain to $S_{J}$ and projecting the range to $S_{J^{\prime}}$ we obtain a map $\theta_{g}: S_{J} \rightarrow S_{J^{\prime}}$. It suffices to show that $\theta_{g}\left(\pi^{r_{J}} S_{J}\right) \subset \pi^{r_{J} J^{\prime}} S_{J^{\prime}}$, that is, that $\theta_{g}\left(S_{J}\right) \subset \pi^{d_{J}-d_{J^{\prime}}} S_{J^{\prime}}$. This is obvious if $d_{J} \leqslant d_{J^{\prime}}$ so we suppose that $d_{J}>d_{J^{\prime}}$. The following proposition then does the trick.

Proposition 10. $\quad \theta_{g}\left(S_{J} \otimes W\right) \subset p^{d_{J}-d_{J^{\prime}}}\left(S_{J^{\prime}} \otimes W\right)$.

Proof. Let $J=\left\{w_{i}\right\}$ with associated numbers $\left\{n_{i}, m_{i}\right\}$ (as in Section 5) and $J^{\prime}=\left\{w_{i}^{\prime}\right\}$ with associated numbers $\left\{n_{i}^{\prime}, m_{i}^{\prime}\right\}$. We may choose $w_{1}$ so that either $n_{1}=0$ or $m_{1}=0$. We suppose that $m_{1}=0$ since the other case is similar. For any $i$ we have $n_{f+i}-n_{i}=\left(\sum_{j=i}^{f+i-1} k_{j}\right)-n f / 2$ so that $F^{f}\left(p^{n_{i}} w_{i}\right)=p^{n_{f+i}+n f / 2} w_{f+i}$ up to multiplication by a $p$-adic unit. Similar statements hold for the $m_{i}, n_{i}^{\prime}$ and $m_{i}^{\prime}$.

For an arbitrary fixed $j$ suppose that the coefficient of $w_{j}^{\prime}$ in $g \cdot p^{n_{1}} w_{1}$ is exactly divisible by $p^{a}$. Now $F^{f}\left(p^{n_{1}} w_{1}\right)$ is divisible by $p^{n_{f+1}+n f / 2}$ so $g . F^{f}\left(p^{n_{1}} w_{1}\right)$ is divisible by $p^{n_{f+1}+n f / 2}$. But $g$ commutes with $F^{f}$ so certainly the coefficient of $w_{f+j}^{\prime}$ in $F^{f} g \cdot p^{n_{1}} w_{1}$ is divisible by $p^{n_{f+1}+n f / 2}$. Hence

$$
a+n_{f+j}^{\prime}-n_{j}^{\prime}+n f / 2 \geqslant n_{f+1}+n f / 2 .
$$

Hence (since $m_{1}=0$ ) we have

$$
a \geqslant n_{j}^{\prime}+n_{f+1}-n_{f+j}^{\prime} \geqslant n_{j}^{\prime}+\left(n_{f+1}+m_{1}\right)-\left(n_{f+j}^{\prime}+m_{j}^{\prime}\right)=n_{j}^{\prime}+d_{J}-d_{J^{\prime}} .
$$

This implies that $\theta_{g}\left(p^{n_{1}} w_{1} \otimes e_{0}\right) \subset p^{d_{J}-d_{J}}\left(S_{J^{\prime}} \otimes W\right)$. In fact, since $g$ is acting only on $H^{n}\left(X_{n}\right)$, we have $\theta_{g}\left(p^{n_{1}} w_{1} \otimes e_{0}\right) \subset p^{d_{J}-d_{J^{\prime}}}\left(H_{J^{\prime}} \otimes W e_{0}\right)$.

Up to $p$-adic units, $p^{m_{i+1}} w_{i+1}=F p^{n_{i}} w_{i} / p^{m}$ and $p^{n_{i+2}} w_{i+2}=F p^{m_{i+1}} w_{i+1} / p^{m-1}$. Similar statements apply with $J$ replaced by $J^{\prime}$. Repeatedly applying $F / p^{m}$ and $F / p^{m-1}$ to the case $i=1$ we obtain

$$
\begin{array}{ll}
\theta_{g}\left(p^{n_{i}} w_{i} \otimes e_{0}\right) \subset p^{d_{J^{-}}-d_{J^{\prime}}}\left(S_{J^{\prime}} \otimes W\right) & \text { for } i \text { odd }, \\
\theta_{g}\left(p^{m_{i}} w_{i} \otimes e_{1}\right) \subset p^{d_{J^{-}}-d_{J^{\prime}}}\left(S_{J^{\prime}} \otimes W\right) & \text { for } i \text { even. }
\end{array}
$$

That takes care of half the basis elements for $S_{J} \otimes W$. But we also have $m_{i}=0$ for some even $i$ or $n_{i}=0$ for some odd $i$ so we may take care of the rest similarly. This is because our basis elements for $S_{J} \otimes W$ come in two $F$-orbits and for each orbit there is a minimum $n_{i}$ or $m_{i}$ equal to zero. 


\section{Some remarks}

(1) There remains the problem of obtaining a reasonable lower bound for the minimal norm of $L$. This leads to several problems. One needs to know the relationship between $M \otimes \mathbb{Z}_{p}$ and $\left(H^{n}\left(X_{n}\right) \otimes H^{1}(E)\right)^{F=p^{m}}$. Are they always equal as in the case $n=1$ ? Is det $M$ a pure power of $p$ before rescaling the intersection pairing? Then one needs a lower bound for the self-intersection number of a cycle in $C^{m} \cap A^{m}$. In the case $n=1$ one divides $\# X_{1}(k)$ by $\# E(k)$ and obtains the bound $2(q-1)$. In the case $n=1$ a lower bound for the minimal norm of $L$ is $2(q-1) / p^{f / 2}$ (since $d_{\max }=f / 2$ ). I do not know what to do for $n>1$.

(2) When $f$ is odd it is easy to adapt the above methods to produce an $R$-stable, $G$-invariant, even integral lattice $L$ in $V$ with $\operatorname{det} L=p^{(\operatorname{rank} L) / 2}$

(3) Remember that $L$ (and $R$, up to conjugacy) depend on the particular choice of $E$ in a fixed $k$-isogeny class. For a given maximal order $R$ in $K$, does every even, unimodular, $R[G]$-stable lattice in $V$ arise from our construction for some choice of $E$ with endomorphism ring $R$ ?

(4) When $n$ is even, primitive codimension $n / 2$ cycles on $X_{n}$ give an irreducible $\mathbb{Q}[G]$-module $V$ of dimension $q^{n+1}-q^{n}+\cdots+q^{3}-q^{2}+q$ as a vector space. The intersection pairing endows $V$ with a $G$-invariant, definite, symmetric bilinear form. Our method breaks down since when $n$ is even the minimal degree of an absolutely irreducible, nontrivial representation of $G$ in characteristic not equal to $p$ is $q^{n+1}-q^{n}+\cdots+q^{3}-q^{2}+q-1[\mathbf{1 5}]$. This is just as well since otherwise we would be able to produce even unimodular lattices of rank not divisible by 8 , which is impossible.

(5) If $p \geqslant n+1$ the reduction $\bmod p$ of $H^{n}\left(X_{n}\right)$ (namely $\left.H_{d R}^{n}\left(X_{n}\right)\right)$ has $(n+1)^{f}$ irreducible $k[G]$-module composition factors, each occurring with multiplicity one.

\section{References}

1. E. BannaI, 'Positive definite unimodular lattices with trivial automorphism groups', Mem. Amer. Math. Soc. 429 (1990) 1-70.

2. N. Dummigan, 'The determinants of certain Mordell-Weil lattices', Amer. J. Math. 117 (1995) $1409-1429$.

3. N. Dummigan, 'Symplectic group lattices as Mordell-Weil sublattices', J. Number Theory 61 (1996) $365-387$.

4. N. D. Elkies, 'Mordell-Weil lattices in characteristic 2: I. Construction and first properties, Internat. Math. Res. Notices 8 (1994) 343-360.

5. N. D. ElkiES, Papers in preparation on Mordell-Well lattices.

6. R. Gow, 'Unimodular integral lattices associated with the basic spin representations of $2 A_{n}$ and $2 S_{n}$, Bull London Math. Soc. 21 (1989) 257-262.

7. R. Gow, 'Even unimodular lattices associated with the Weil representation of the finite symplectic group', J. Algebra 122 (1989) 510-519.

8. B. H. Gross, 'Group representations and lattices', J. Amer. Math. Soc 3 (1990) 929-960.

9. A. Grothendieck, 'Standard conjectures on algebraic cycles', Algebraic geometry, Bombay 1968 (Oxford University Press, 1969) 193-199.

10. B. HaAstert and J.C. JANTzEn, 'Filtrations of the discrete series of $S L_{2}(q)$ via crystalline cohomology', J. Algebra 132 (1990) 77-103.

11. R. Hotta and K. Matsui, 'On a lemma of Tate-Thompson', Hiroshima Math. J. 8 (1978) 255-268.

12. L. Illusie, 'Report on crystalline cohomology', Proc. Sympos. Pure Math. 29 (1975) 459-478.

13. T. Katsura and T. Shioda, 'On Fermat varieties', Tôhoku Math. J. 31 (1979) 97-115.

14. N. Katz, 'On the intersection matrix of a hypersurface', Ann. Sci. École Norm. Sup (4) t.2 (1969) 583-598.

15. V. LANDAZURi AND J. Seitz, 'On the minimal degrees of projective representations of finite Chevalley groups', J. Algebra 32 (1974) 418-443.

16. B. Mazur, 'Frobenius and the Hodge filtration', Bull. Amer. Math. Soc. 78 (1972) 653-667.

17. B. Mazur, 'Frobenius and the Hodge filtration (estimates)', Ann. of Math. 98 (1973) 58-95. 
18. J. S. Milne, 'Values of zeta functions of varieties over finite fields', Amer. J. Math. 108 (1986) $297-360$.

19. A. OGus, 'Griffiths transversality in crystalline cohomology', Ann. of Math. 108 (1978) 395-419.

20. T. SHIODA, 'Mordell-Weil lattices and sphere-packings', Amer. J. Math. 113 (1991) 931-948.

21. J. TATE, 'Algebraic cycles and poles of zeta functions', Arithmetical algebraic geometry, (ed. O. F. G. Schilling; Harper and Row, New York, 1965) 93-110.

22. J. TATE, 'Endomorphisms of abelian varieties over finite fields', Invent. Math. 2 (1966) 134-144.

23. J. G. ThOMPSON, 'Finite groups and even lattices', J. Algebra 38 (1976) 523-524.

24. P. H. TIEP, 'The automorphism groups of some Mordell-Weil lattices', Izv. Akad. Nauk SSSR Ser. Mat. 56 (1992) 509-537.

25. P. H. TIEP, 'Basic spin representations of $2 S_{n}$ and $2 A_{n}$ as globally irreducible representations', Arch. Math. 64 (1995) 103-112.

26. P. H. TIEP, 'Weil representations as globally irreducible representations', Math. Nachr. 184 (1997) 313-327.

27. W. C. Waterhouse, 'Abelian varieties over finite fields', Ann. Sci. École Norm. Sup. (4) t.2 (1969) $521-560$.

28. A. WeIL, 'Numbers of solutions of equations in finite fields', Bull Amer. Math. Soc. 55 (1949) 497-508.

Department of Mathematics

University of Michigan

Ann Arbor

Michigan 48109

USA

Current address:

Department of Mathematical Sciences

Northern Illinois University

De Kalb

Illinois 60115

USA 